\title{
Effects of tyrosol and farnesol on Candida albicans biofilm
}

\author{
SARRA SEBAA $^{1,2}$, ZAHIA BOUCHERIT-OTMANI ${ }^{2}$ and PHILIPPE COURTOIS ${ }^{1}$ \\ ${ }^{1}$ Laboratory of Physiology and Pharmacology, Université Libre de Bruxelles, B-1070 Brussels, Belgium; \\ ${ }^{2}$ Laboratory of Antibiotics and Antifungals, Physico-Chemistry, Synthesis and Biological Activity, \\ University of Tlemcen, 13000 Tlemcen, Algeria
}

Received September 17, 2018; Accepted February 13, 2019

DOI: $10.3892 / \mathrm{mmr} .2019 .9981$

\begin{abstract}
The present in vitro study examined the effects of the quorum-sensing molecules farnesol and tyrosol on the development of Candida albicans biofilm in order to elucidate their role as novel adjuvants in oral hygiene. The investigation was conducted in C.albicans ATCC 10231 and C.albicans isolates from dentures and was performed in flat-bottomed 96-well polystyrene plates. Yeast growth and their capacity to form biofilms were evaluated following 24 and $48 \mathrm{~h}$ incubations at $37^{\circ} \mathrm{C}$ in Sabouraud broth supplemented with 0.001-3 mM farnesol and/or 1-20 mM tyrosol. Yeast growth was assessed by turbidimetry and biofilms were quantitated by crystal violet staining, under aerobic and anaerobic conditions. The viability of the fungal cells was controlled by the culture of planktonic cells and by examination of the biofilms using fluorescence microscopy following staining with fluorescein diacetate and ethidium bromide. Farnesol at $3 \mathrm{mM}$ exerted a stronger action when added at the beginning of biofilm formation ( $>50 \%$ inhibition) than when added to preformed biofilms ( $<10 \%$ inhibition). Similarly, tyrosol at $20 \mathrm{mM}$ had a greater effect on biofilm formation ( $>80 \%$ inhibition) than on preformed biofilms ( $<40 \%$ inhibition). Despite significant reductions in attached biomass, yeast growth varied little in the presence of the investigated molecules, as corroborated by the turbidimetry, culture of supernatants on solid culture medium followed by counting of colony-forming units and viability tests using fluorescence microscopy. At the highest tested concentration, the molecules had a greater effect during the initial phases of biofilm formation. The effect of farnesol during anaerobiosis was not significantly different from that observed during aerobiosis, unlike that of tyrosol during anaerobiosis, which exhibited slightly reduced yeast biofilm inhibition. In conclusion, the present study demonstrated the specific anti-biofilm effect, independent of fungicidal
\end{abstract}

Correspondence to: Dr Philippe Courtois, Laboratory of Physiology and Pharmacology, Université Libre de Bruxelles, Route de Lennik 808, B-1070 Brussels, Belgium

E-mail: philippe.courtois@ulb.ac.be

Key words: Candida, biofilm, dentures, farnesol, tyrosol, quorum sensing, yeast or fungistatic action, of farnesol and tyrosol, as tested in C. albicans ATCC 10231 and 6 strains isolated from dentures. Prior to suggesting the use of these molecules for preventive purposes in oral hygiene, further studies are required in order to clarify the metabolic pathways and cellular mechanisms involved in their antibiofilm effect, as well as the repercussions on the oral microbiome.

\section{Introduction}

Quorum-sensing (QS) mechanisms allow microorganisms to adapt different biological functions to the environment according to their population density via the secretion of self-inducing signalling molecules. These quorum-sensing molecules (QSMs) are able to suppress or activate gene expression (1-3). QSMs have been described in bacteria and in yeast such as Candida, a well-known contaminant for dentures. Candida-secreted QSMs induce phenotypic adaptations that include morphological changes (4), secretion of virulence factors (5) and biofilm formation (6). The following four types of QSMs have been identified in Candida albicans: i) 2-phenylethanol and tryptophol derived from phenylalanine and tryptophan, respectively (7); ii) the MARS molecule of unknown structure (8); iii) farnesol (9) and its derivative farnesoic acid (10); and iv) tyrosol (11). Farnesol and tyrosol are the two molecules that have been the most widely studied to date.

Farnesol is a metabolite of the mevalonate/sterol synthesis pathway in C.albicans. In suspension, Candida blastoconidia produce farnesol up to a maximal concentration of $10-50 \mu \mathrm{M}$ in the stationary phase (9). Candida growth in liquid culture medium is correlated with an increase in resistance to oxidative stress by the expression of superoxide dismutase and catalase, suggesting a link with QSMs (12). Furthermore, farnesol accumulation blocks the yeast-hyphal transition of C. albicans at high cell densities without blocking the elongation of pre-existing hyphae (13) or influencing cell growth rates (9). A previous study suggested that exogenous farnesol, which was tested on a $C$. albicans strain that does not produce endogenous farnesol, suppresses hyphal formation by inducing morphological changes in the yeast cell wall and by suppression of the expression of aspartyl proteinases (14). Farnesol prevents $C$. albicans biofilm formation (15). Numerous stages of biofilm development are influenced by farnesol, including cell adhesion to substrates, mature biofilm architecture, and cell dispersion from the biofilm. Due to the effect of farnesol 
on morphology, and the importance of morphology in biofilm formation, it has been suggested that exogenous farnesol affects biofilm development by repressing hyphal formation and the expression of genes specific to filamentation (6). A number of studies have expressed an interest in using this molecule in therapeutics or hygiene (3,15-17). Combining farnesol with fluconazole reduces the thickness of $C$. albicans biofilm and the minimal inhibitory concentration of fluconazole, indicating that farnesol inhibits the development of fluconazole resistance in $C$. albicans strains known to be resistant to this antifungal (18). A similar effect was also demonstrated on C. dubliniensis (19). The significant synergy between farnesol and three different antifungals (micafungin, fluconazole and amphotericin B) has beneficial effects against $C$. albicans biofilm (20).

Tyrosol, derived from tyrosine, accelerates the formation of germ tubes without compensating for the effect of farnesol in blocking germination (21). This molecule is considered a minor QSM, whose influence is observed only when the concentration of farnesol is low or absent in the environment (5). At micromolar concentrations, tyrosol stimulates hyphal production during the early stages of $C$. albicans biofilm formation (22). In the millimolar range, exogenous tyrosol has been reported to inhibit the formation of biofilms (23). The combination of tyrosol with other antifungals (amphotericin B, itraconazole and fluconazole) has a synergistic effect on $C$. albicans and C. tropicalis biofilms (23).

An improved understanding of the action of these two QSMs may lead to the design of novel antifungal strategies that target Candida biofilm formation and development, particularly for prophylactic approaches in oral hygiene. Current antifungals should be reserved for the treatment of patients with infections, as these drugs are less active against yeasts organised in biofilms (17-20). Furthermore, antifungal use on yeast-colonised dentures in the oral environment promotes the emergence of resistant strains (17-20). From this perspective, the present study aimed to compare the effects of both QSMs (farnesol and tyrosol) individually on C. albicans biofilm.

\section{Materials and methods}

Preparation of QSMs. The two QSMs (tyrosol and farnesol) were purchased from Sigma-Aldrich (Merck KGaA, Darmstadt, Germany). Farnesol is insoluble in water, unlike tyrosol (solubility threshold, $25.3 \mathrm{mg} / \mathrm{ml}$ ); therefore, a stock solution of farnesol was produced by transferring $50 \mu 1$ reagent (trans,trans-3,7,11-trimethyl-2,6,10-dodecatriene-1-ol, $\mathrm{C}_{15} \mathrm{H}_{26} \mathrm{O}$, PM: $222.37 \mathrm{~g} / \mathrm{mol}$, density: $0.879 \mathrm{~g} / \mathrm{ml}$ ) to $6.275 \mathrm{ml}$ $1 \%(\mathrm{v} / \mathrm{v})$ DMSO (24 mM). Subsequent dilutions were made in $1 \%$ DMSO and incorporated into the reaction medium (final concentrations, $0.001-3 \mathrm{mM}$ farnesol in $0.1 \%$ DMSO). The farnesol controls contained DMSO at a similar concentration to that present in the assays $(0.1 \%)$. Tyrosol [4-(2-hydroxyethyl)phenol, $\mathrm{C}_{8} \mathrm{H}_{10} \mathrm{O}_{2}$, PM: $138.16 \mathrm{~g} / \mathrm{mol}$ ] was brought to final concentrations of $1-20 \mathrm{mM}$ from a $22-\mathrm{mM}$ stock solution in Sabouraud broth.

Microorganisms. The investigation was conducted in C. albicans ATCC 10231 (Culti-loops $^{\mathrm{TM}}$; Thermo Fisher Scientific, Inc., Waltham, MA, USA), a reference strain often used to test antifungals (24), and six clinical strains of C. albicans (provided by Oral Biology Unit, Laboratory of Physiology and Pharmacology, Université Libre de Bruxelles, Brussels, Belgium), which were isolated from dentures using sterile cotton swabs (Eurotubo ${ }^{\circledR}$; Deltalab, Barcelona, Spain). Yeast were aerobically grown at $37^{\circ} \mathrm{C}$ for $24 \mathrm{~h}$ in Sabouraud broth (CM147; Oxoid; Thermo Fisher Scientific, Inc.) or on solid Sabouraud agar (BD Diagnostics, Erembodegem, Belgium). Clinical isolates were identified according to the appearance of their colonies on CHROMagar ${ }^{\mathrm{TM}}$ medium (BD Diagnostics), by chlamydoconidia formation on rice extract agar containing polysorbate 80 (BD Diagnostics) and using the API yeast identification system (bioMérieux, Marcy-l'Etoile, France). All in-vitro studies were conducted in a third subculture on Sabouraud agar, from which the yeasts were transferred into Sabouraud broth. Candida suspensions were adjusted to $10^{7}$ yeast cells (blastoconidia) per millilitre by dilution following counting in a Thoma cell counting chamber (Marienfeld, Lauda-Königshofen, Germany). A number of the experiments were conducted under anaerobic conditions that were generated by Anaerogen ${ }^{\mathrm{TM}}$ sachets placed in anaerobic 3.5-1 jars (Oxoid; Thermo Fisher Scientific, Inc.).

Biofilm production and quantitation. Candida biofilm formation was achieved in flat-bottomed 96 -well polystyrene plates (12x8-well columns; CellStar ${ }^{\circledR}$; Greiner Bio-One International $\mathrm{GmbH}$, Neuburg, Germany) for both the reference and clinical strains. Each experimental condition included eight replicates distributed in one column of the microplate. In addition to the different concentrations tested, each experiment always included controls without QSMs and sterility controls without cell suspension to attest to the absence of accidental contamination during handling. The minimal inhibitory concentration of both investigated QSMs (farnesol and tyrosol) was estimated by the evaluation of turbidimetric growth in broth and by quantitation of attached biomass in the reactive wells. Yeast growth and their capacity to form a biofilm were assessed after $24 \mathrm{~h}$ at $37^{\circ} \mathrm{C}$ in liquid Sabouraud medium with increasing concentrations of farnesol (0.001-3 mM) and tyrosol (1-20 mM). Each well (except those for the sterility controls) initially contained $0.25 \times 10^{6}$ blastoconidia suspended in $25 \mu \mathrm{l}$ Sabouraud broth (final volume in each well, $250 \mu \mathrm{l}$ ). The contents of the plate were mixed for $5 \mathrm{~min}$ at room temperature using a plate shaker (IKA ${ }^{\mathrm{TM}}$, Boutersem, Belgium) and then incubated at $37^{\circ} \mathrm{C}$ for $24 \mathrm{~h}$. The following day, yeast growth was photometrically assessed at $600 \mathrm{~nm}$ using a microplate reader (Packard SpectraCount ${ }^{\mathrm{TM}}$; PerkinElmer, Inc., Waltham, MA, USA). This turbidimetric evaluation did not differentially evaluate free cells and those attached to the bottom of the wells. The assay data were compared to the controls. Following the opacimetric reading at $24 \mathrm{~h}$, the biofilms were quantitated by crystal violet staining according to a method adapted from a previously published study (25). Briefly, the Sabouraud broth was carefully aspirated using a multi-channel pipette (VWR International, Leuven, Belgium) and the microplate wells were gently (considering the fragility of biofilm structures) washed three times with $250 \mu \mathrm{l}$ saline to remove the non-adherent cells. The attached biomass was fixed with $250 \mu$ l methanol (Sigma-Aldrich; Merck KGaA) for $15 \mathrm{~min}$ at room temperature and then stained with $250 \mu 12 \%$ Hucker crystal violet solution 
(8 g crystal violet in $80 \mathrm{ml} 95 \%$ ethanol, plus $3.2 \mathrm{~g}$ ammonium oxalate in $320 \mathrm{ml}$ distilled water) at room temperature for a further $5 \mathrm{~min}$. After gentle rinsing with running tap water and drying with a hair dryer, the coloured biomass attached to the walls of the microplate wells was solubilised with $250 \mu 12 \mathrm{M}$ acetic acid for $30 \mathrm{~min}$, and the absorbance was measured at 600 nm using a Packard SpectraCount ${ }^{\mathrm{TM}}$ microplate reader. All samples with an absorbance value $>2.000$ were diluted 10 -fold in $2 \mathrm{M}$ acetic acid. The coefficient of variation for the Packard SpectraCount ${ }^{\mathrm{TM}}$ microplate reader is $<5 \%$.

In a number of the experiments, the culture medium was diluted 100- and 1,000-fold in saline following the 24-h incubation and prior to biofilm quantitation. A $20-\mu l$ aliquot of each dilution was seeded onto Sabouraud agar, containing $0.4 \mathrm{~g} / 1$ chloramphenicol and $0.04 \mathrm{~g} / 1$ gentamycin, in order to count the colony forming units (CFUs) after $48 \mathrm{~h}$ at $37^{\circ} \mathrm{C}$ using an e-Count ${ }^{\mathrm{TM}}$ colony counter (Heathrow Scientific, Vernon Hills, IL, USA). Subsequently, the effect of the QSMs on Candida biofilm was also studied over a longer period of 48-72 h, with the addition of the molecules prior to biofilm formation or following a $24-\mathrm{h}$ incubation at $37^{\circ} \mathrm{C}$. Under this last condition, the culture medium was renewed after $24 \mathrm{~h}$.

Fluorescent staining. Fresh solutions of fluorescein diacetate (5 $\mathrm{mg} / \mathrm{ml}$ in acetone) and ethidium bromide $(5 \mathrm{mg} / \mathrm{ml}$ in phosphate-buffered saline, pH 7.5) (Sigma-Aldrich; Merck KGaA) were separately diluted 100-fold in phosphate-buffered saline and then mixed at a 1:1 ratio. The fluorescent reagent and Candida biofilm suspended in saline washing liquid were mixed at a 1:1 ratio, and subsequently incubated for $15 \mathrm{~min}$ at $37^{\circ} \mathrm{C}$ prior to microscopic examination (Leica DM2000; Leica Microsystems, Inc., Buffalo Grove, IL, USA). Green fluorescence was considered as living cells and orange staining as non-viable cells.

Statistical analysis. The data are presented as a percentage following division of each experimental value by the arithmetic mean of the paired control values obtained under the same conditions. Considering the intra-assay control as $100 \%$ eliminated the bias of inter-assay variability. The data are presented as the mean \pm standard error of the mean (SEM), unless otherwise indicated. Data management and statistical tests (mean, standard deviation, SEM, Kolmogorov-Smirnov test, unpaired Student's t-test, and ANOVA supplemented with a Dunnett's multiple comparison post-hoc test) were performed using GraphPad Prism software, version 7.01 for Windows (GraphPad Software, Inc., La Jolla, CA, USA). P<0.05 was considered to indicate a statistically significant difference.

\section{Results}

Effect of farnesol and tyrosol on Candida growth. DMSO used to solubilize farnesol did not inhibit the growth of C. albicans ATCC 10231 at final concentrations of $0.1-2.5 \%$ (data not shown). Neither farnesol molecule nor that of tyrosol, despite the concentration, modified the $\mathrm{pH}$ of the reaction medium (data not shown). Figs. 1 and 2 present the effect of different concentrations of farnesol (0.001-3 mM) and tyrosol (1-20 mM) on the growth of C. albicans ATCC 10231 and six clinical strains isolated from dentures, respectively. Following

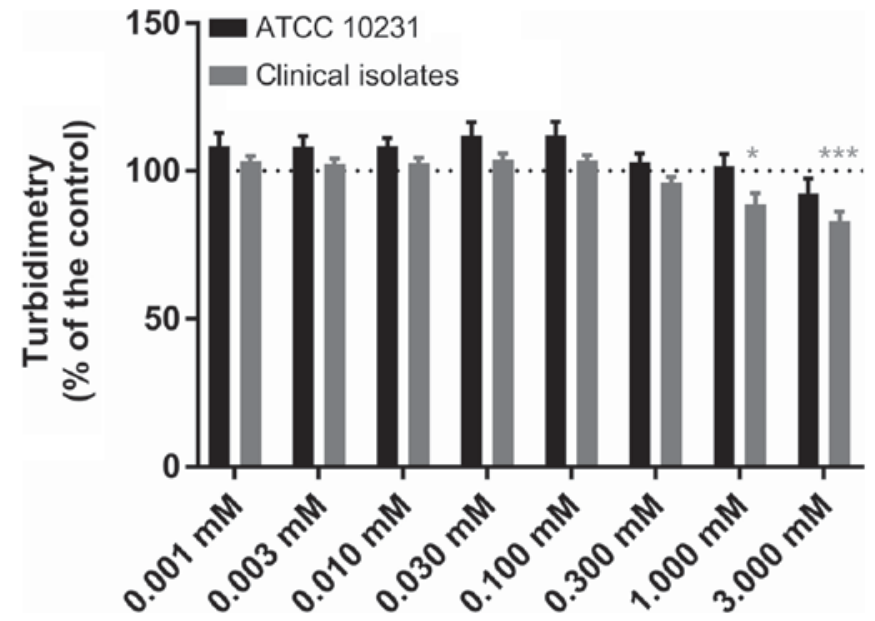

Figure 1. Effect of farnesol on Candida growth. The effect of farnesol (0.001-3 mM) on the turbidimetric growth of C. albicans in ATCC 10231 and clinical strains. The dotted lines indicate the $100 \%$ standardised control value. Data are presented as the mean \pm standard error of the mean; $n=6$ independent experiments with 8 replicates for each condition. ${ }^{*} \mathrm{P}<0.05$ and **** $\mathrm{P}<0.001$ vs. control.

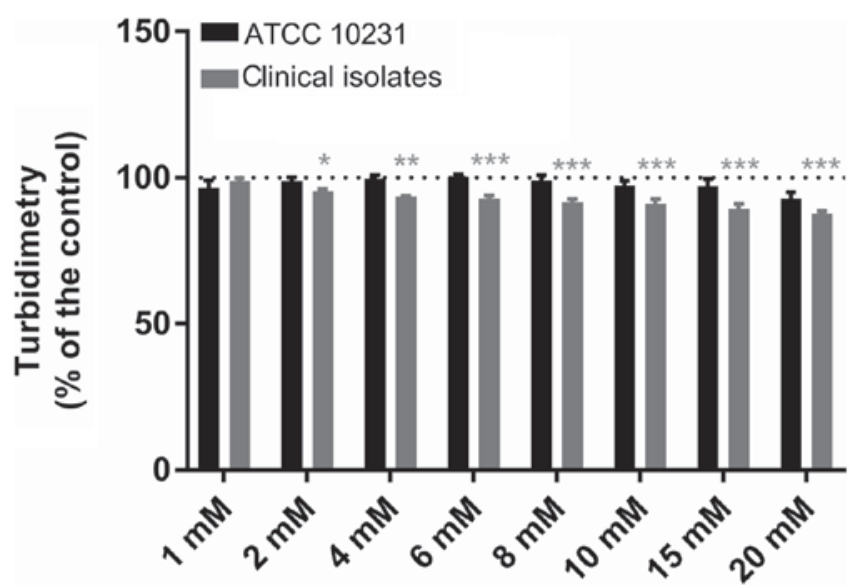

Figure 2. Effect of tyrosol on Candida growth. The effect of tyrosol (1-20 mM) on the turbidimetric growth of C.albicans in ATCC 10231 and clinical strains. The dotted lines indicate the $100 \%$ standardised control value. Data are presented as the mean \pm standard error of the mean; $n=6$ independent experiments with 8 replicates for each condition. ${ }^{*} \mathrm{P}<0.05,{ }^{* * *} \mathrm{P}<0.01$ and ${ }^{* * *} \mathrm{P}<0.001$ vs. control.

a 24-h incubation at $37^{\circ} \mathrm{C}$, the turbidity of the culture medium in all control wells ( $\mathrm{n}=96$ in six independent experiments) represented an absorbance at $600 \mathrm{~nm}$ of $0.735 \pm 0.010$ for the reference strain and $0.885 \pm 0.024$ for the clinical strains. The replicates in each series of 8 control wells exhibited a coefficient of variation of $<9 \%$ for turbidimetric measurements. Farnesol (Fig. 1) did not modify the observed turbidity (Dunnett's, NS) at concentrations of $1 \mu \mathrm{M}-0.3 \mathrm{mM}(\mathrm{n}=6)$. The absorbance at $600 \mathrm{~nm}$ following a 24-h incubation at $37^{\circ} \mathrm{C}$ varied from $103.0 \pm 2.9$ to $112.1 \pm 4.5 \%$ of that observed in the paired controls for the ATCC strain and from 96.0 2.0 to $103.9 \pm 2.0 \%$ for the isolates. A $1 \mathrm{mM}$ concentration of farnesol slightly reduced the turbidimetric growth of the clinical strains, while it did not modify growth of the ATCC strain

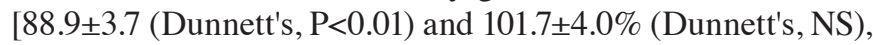




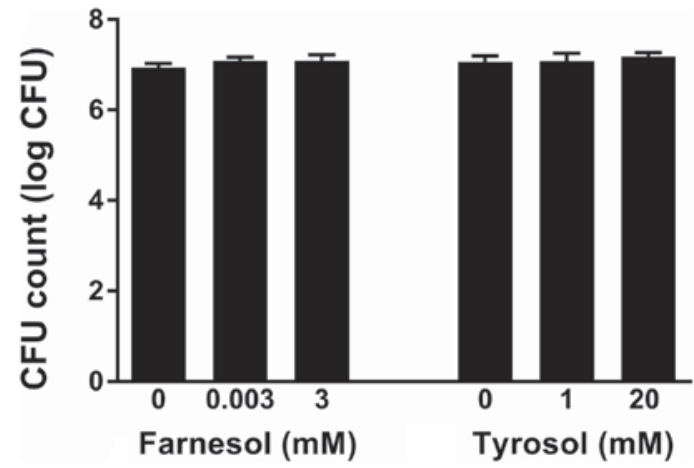

Figure 3. Effect of QSMs on the number of Candida CFUs. The effect of farnesol (0.001-3 mM) and tyrosol (1-20 mM) on the number of Candida CFUs measured in the culture supernatants following a 24-h incubation at $37^{\circ} \mathrm{C}$ (ATCC 10231 strain). The CFU count is expressed as the logarithm. QSMs, quorum-sensing molecules; CFUs, colony forming units.

respectively]. Similarly, $3 \mathrm{mM}$ farnesol reduced the turbidimetric growth of all strains, but only significantly for the clinical strains $[83.0 \pm 3.3$ (Dunnett's, $\mathrm{P}<0.001$ ) in the isolates and $92.4 \pm 5.0 \%$ (Dunnett's, NS) in the reference strain]. Tyrosol (Fig. 2) at concentrations of 1-20 mM did not affect the growth of the ATCC 10231 strain (Dunnett's, NS), despite a non-significant reduction of $8.1 \pm 2.2 \%$ at $20 \mathrm{mM}(\mathrm{n}=6)$. Thus, the absorbance at $600 \mathrm{~nm}$ following a $24-\mathrm{h}$ incubation at $37^{\circ} \mathrm{C}$ varied from $92.9 \pm 2.2$ to $100.4 \pm 4.5 \%$ of that observed in the paired controls. By contrast, for the clinical strains, 2-20 mM tyrosol slightly and gradually reduced the turbidimetric growth from 4.6 \pm 0.7 (Dunnett's, $\mathrm{P}<0.05$ ) to $12.3 \pm 0.9 \%$ (Dunnett's, $\mathrm{P}<0.01)$. However, in the ATCC 10231 strain culture supernatants diluted 50,000 times following a $24-\mathrm{h}$ incubation at $37^{\circ} \mathrm{C}$ in the presence of QSMs, the CFU count was not significantly different with either farnesol (ANOVA, $\mathrm{P}=0.4921$ ) or tyrosol $(\mathrm{P}=0.8202)$ relative to their paired control. The number of CFUs expressed as logarithms is presented in Fig. 3.

Effect of farnesol and tyrosol on Candida biofilm formation. Figs. 4 and 5 present the results of the evaluation of the biomass attached to the inner wall of the microplate wells following a $24-\mathrm{h}$ incubation at $37^{\circ} \mathrm{C}$ in the presence of each investigated QSM. Following crystal violet staining and 10 -fold dilution, the controls exhibited an absorbance at 600 $\mathrm{nm}$ of $0.539 \pm 0.048$ ( $\mathrm{n}=6$ independent experiments) for the reference strain and $0.668 \pm 0.072(\mathrm{n}=6$ independent experiments) for the clinical strains. The replicates in each series of 8 control wells exhibited a coefficient of variation of $<30 \%$ (10.9-29.5\%) for biofilm quantitation. Fig. 4 demonstrates a statistically significant inhibitory effect of farnesol at 1 and $3 \mathrm{mM}$ on the formation of biofilms by both the reference strain and the clinical strains (Dunnett's, $\mathrm{P}<0.001$ ). At a concentration of $3 \mathrm{mM}$, the attached biomass averaged only $10.5 \pm 4.6(n=6)$ and $27.8 \pm 4.5 \%(n=6)$ of the paired controls for the ATCC strain and the clinical isolates, respectively. Fig. 5 presents the effect of different tyrosol concentrations between 1 and $20 \mathrm{mM}$ on biofilm formation by both C. albicans ATCC 10231 and the clinical strains. The data obtained in the presence of tyrosol reveal a fixed biomass that was inversely proportional to the tyrosol concentration when it was $\geq 4 \mathrm{mM}$, and became significant at $6 \mathrm{mM}$ in the reference

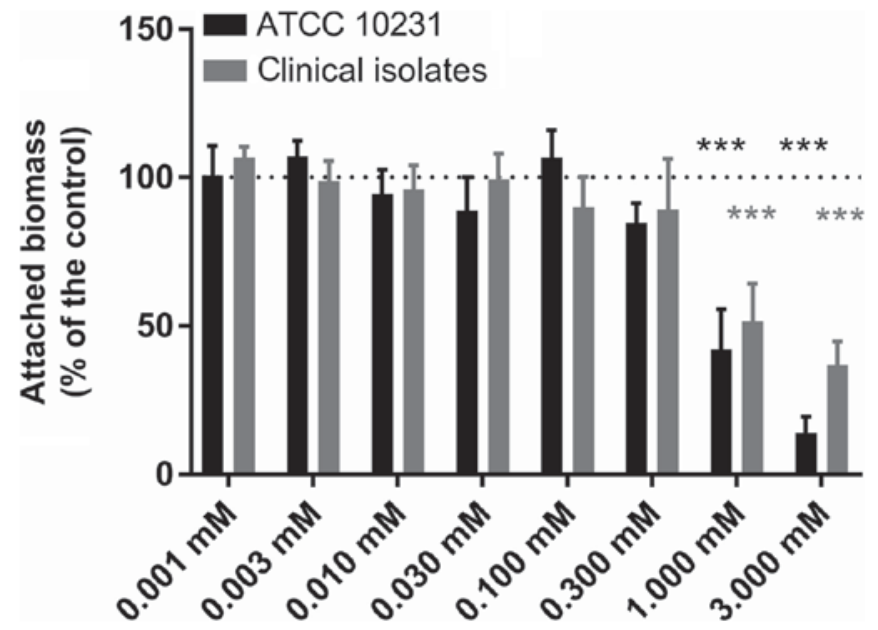

Figure 4. Effect of farnesol on Candida biofilm. The effect of farnesol (0.001-3 mM) on biofilm formation by C. albicans in ATCC 10231 and clinical strains. Dotted lines indicate the $100 \%$ standardised control value. Data are presented as the mean \pm standard error of the mean; $n=6$ independent experiments with 8 replicates for each condition. ${ }^{* * * *} \mathrm{P}<0.001 \mathrm{vs}$. control.

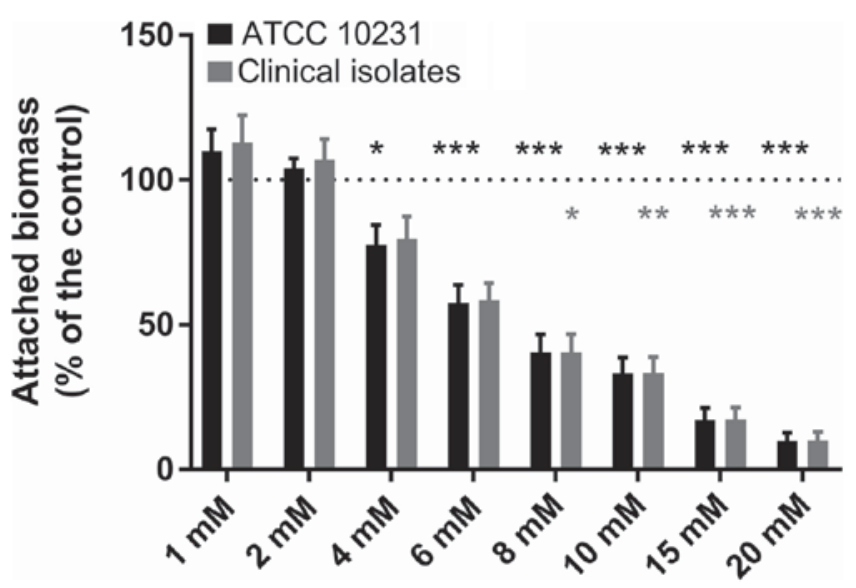

Figure 5. Effect of tyrosol on Candida biofilm. The effect of tyrosol (1-20 mM) on biofilm formation by $C$. albicans in ATCC 10231 and clinical strains. The dotted lines indicate the $100 \%$ standardised control value. Data are presented as the mean \pm standard error of the mean; $n=6$ independent experiments with 8 replicates for each condition. ${ }^{*} \mathrm{P}<0.05,{ }^{* * *} \mathrm{P}<0.01$ and ${ }^{* * *} \mathrm{P}<0.001$ vs. control.

strain (Dunnett's, $\mathrm{P}<0.001$ ) and at $8 \mathrm{mM}$ in the clinical strains (Dunnett's, P<0.05). At $20 \mathrm{mM}$ tyrosol, the amount of biofilm was only $9.9 \pm 2.8(n=6)$ and $10.0 \pm 2.9 \%(n=6)$, respectively, of the mean value of the paired controls.

The effects of farnesol and tyrosol on biofilm formation were evaluated simultaneously under both aerobic and anaerobic conditions (Figs. 6 and 7). Notably, there was a significant reduction in biofilm production $(63.2 \%)$ in anaerobiosis compared with that observed under aerobic conditions (Fig. 6) (unpaired Student's t-test, $\mathrm{P}<0.0001$ ); while the reduction observed in turbidimetric growth in anaerobiosis was only $8.2 \%$ (non-Gaussian distribution, Mann-Whitney test, $\mathrm{P}<0.0001)$. The data reported in Fig. 7 confirms the effects of QSMs on the production of biofilm both aerobically and anaerobically. A $3 \mathrm{mM}$ concentration of farnesol exerted similar effects despite the $\mathrm{O}_{2}$ level; the residual attached 


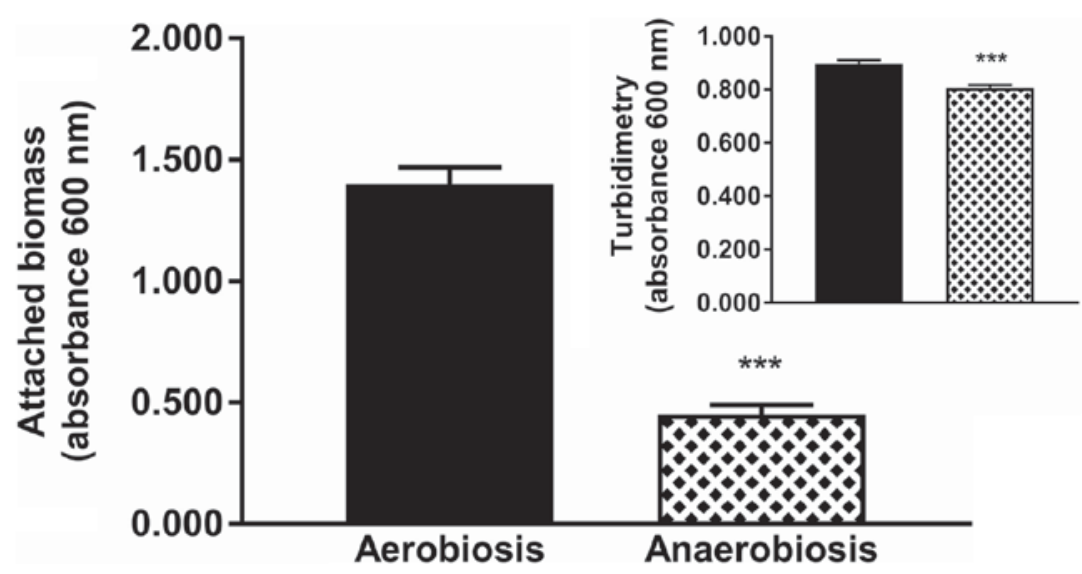

Figure 6. Yeast biofilm production in aerobiosis compared to anaerobiosis. The effect of anaerobiosis on C. albicans ATCC 10231 biofilm formation (n=16 in 2 independent experiments). ${ }^{* * *} \mathrm{P}<0.001$ vs. Aerobiosis group.

biomass was $44.6 \pm 3.0 \%$ in aerobiosis, whereas in anaerobiosis it was $37.8 \pm 3.8 \%$. Statistical analysis revealed a Gaussian distribution of the data, and the result of the Student's t-test was non-significant $(\mathrm{P}=0.1720)$. A $20 \mathrm{mM}$ concentration of tyrosol reduced the attached biomass under aerobic conditions (fixed residual biomass, $8.2 \pm 0.8 \%$ of the matched control) more than in anaerobiosis $(17.5 \pm 2.0 \%)$. Statistical analysis demonstrated a non-Gaussian distribution of the data in anaerobiosis, and a significant non-parametric Mann-Whitney test $(\mathrm{P}=0.0002)$ indicated a greater inhibitory effect of $20 \mathrm{mM}$ tyrosol in aerobiosis than in anaerobiosis. Lower concentrations of farnesol $(0.003 \mathrm{mM})$ or tyrosol $(1 \mathrm{mM})$ resulted in no significant changes in attached biomass (ANOVA and Kruskal-Wallis test, NS) under anaerobic compared to aerobic conditions.

The viability of the fungal cells in the presence of farnesol or tyrosol, already demonstrated by culturing the planktonic cells, was confirmed by fluorescent microscopic examination of the biofilms following staining with fluorescein diacetate, which stains the living cells, and with ethidium bromide, which stains the dead cells. A similar assay conducted on C.albicans ATCC 10231 blastoconidia prior to and following a 30 -min incubation at $80^{\circ} \mathrm{C}$ revealed $100 \%$ live and $100 \%$ dead cells, respectively. Fig. 8A and B demonstrate that there was $>95 \%$ living planktonic Candida cells following incubation at $37^{\circ} \mathrm{C}$ for $30 \mathrm{~min}$, and $100 \%$ dead planktonic cells (C.albicans ATCC 10231) following incubation at $85^{\circ} \mathrm{C}$ for $30 \mathrm{~min}$, assuring the reliability of the reagents. Fig. $8 \mathrm{C}-\mathrm{F}$ presents the characteristics of the $C$. albicans ATCC 10231 biofilm formed in the presence of $3 \mathrm{mM}$ farnesol (Fig. 8D) or $20 \mathrm{mM}$ tyrosol (Fig. 8F) in comparison to their respective controls (Fig. 8C and E). The microscopic observation revealed that $>95 \%$ of the fungal cells (blastoconidia and filaments) were alive following contact with QSMs as compared to their respective controls. Notably, there was a scarcity of filaments in the biofilms following incubation with $3 \mathrm{mM}$ farnesol or $20 \mathrm{mM}$ tyrosol.

Effect of farnesol and tyrosol on Candida pre-formed biofilms. To investigate the effects of QSMs in contact with pre-formed biofilms, the incubation time was extended to $72 \mathrm{~h}$, with renewal of the culture medium at $24 \mathrm{~h}$. Under these conditions and in the absence of QSMs, the attached biomass increased

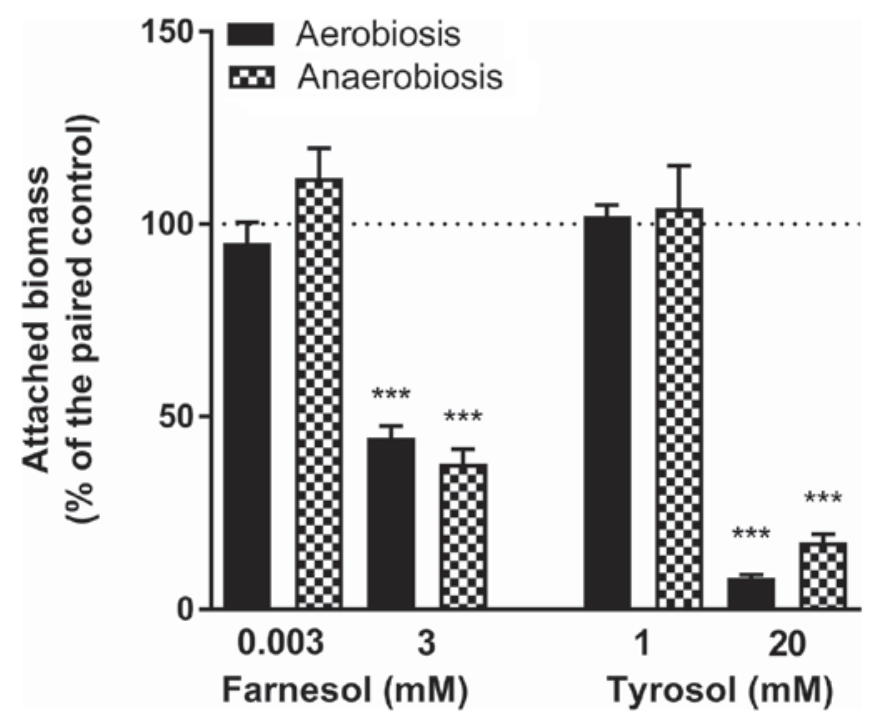

Figure 7. Effect of anaerobiosis on biofilm inhibition by QSMs. The effect of farnesol $(0.003$ and $3 \mathrm{mM})$ and tyrosol $(1$ and $20 \mathrm{mM})$ on biofilm formation under aerobic and anaerobic conditions ( $\mathrm{n}=16$ in 2 independent experiments). QSM, quorum-sensing molecule. ${ }^{* * *} \mathrm{P}<0.001$ vs. control.

over time, as shown in Fig. 9. In a set of six experiments on C. albicans ATCC 10231, the increase in attached biomass at 24 and $48 \mathrm{~h}$ following renewal of the culture medium averaged $170.6 \pm 25.0$ and $236.7 \pm 46.6 \%$ of the paired values, respectively, which was observed prior to the medium change. Following the addition of QSMs at the beginning of biofilm formation (Fig. 10), the biomass attached after $24 \mathrm{~h}$ (expressed as a percentage of that obtained with the paired control) was reduced significantly (Dunnett's, $\mathrm{P}<0.001$ ) in the presence of $3 \mathrm{mM}$ farnesol $(44.6 \pm 3.0 \%, \mathrm{n}=6)$, but remained unchanged in the presence of $0.003 \mathrm{mM}$ farnesol $(90.5 \pm 5.2 \%, \mathrm{n}=6)$. Similarly, tyrosol at $20 \mathrm{mM}$ significantly reduced (Dunnett's, $\mathrm{P}<0.001)$ the formation of biofilm $(8.2 \pm 0.8 \%, \mathrm{n}=6)$, but had no effect at $1 \mathrm{mM}(102.1 \pm 2.9 \%, \mathrm{n}=6)$. Fig. 10 presents the amount of attached biomass observed when the QSMs were added to pre-formed biofilms. When adding $0.003 \mathrm{mM}$ vs. $3 \mathrm{mM}$ farnesol to the pre-formed biofilms, the biomass attached after $24 \mathrm{~h}$ was $106.1 \pm 8.4$ (NS) and $96.3 \pm 10.3 \%$, respectively, of the paired controls, and the biomass attached after $48 \mathrm{~h}$ 
A

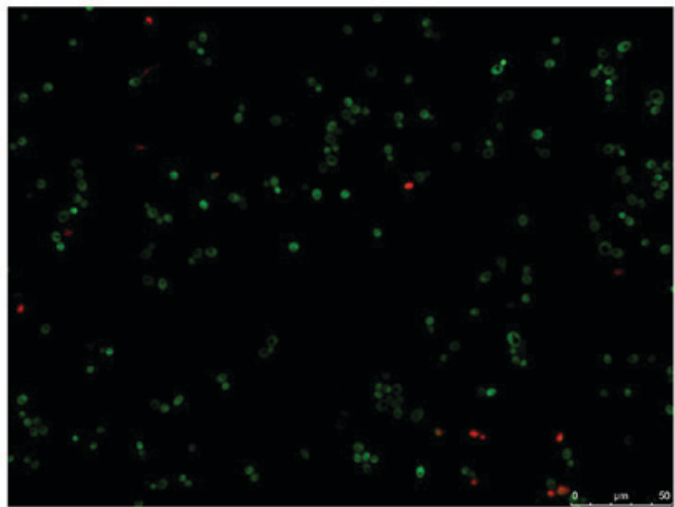

$\mathrm{C}$

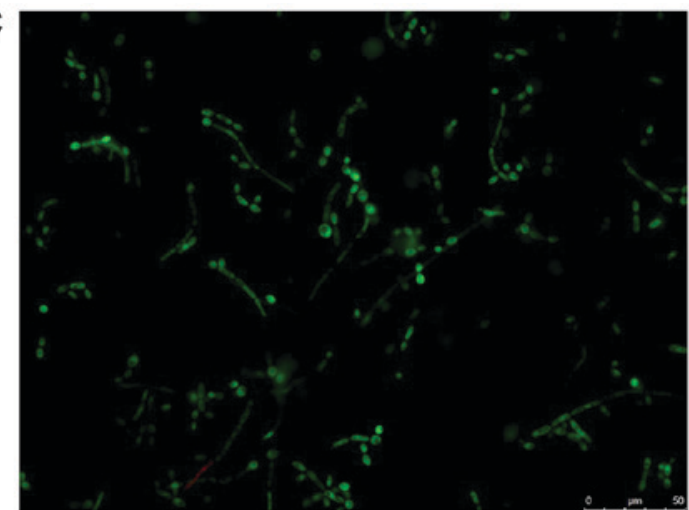

E

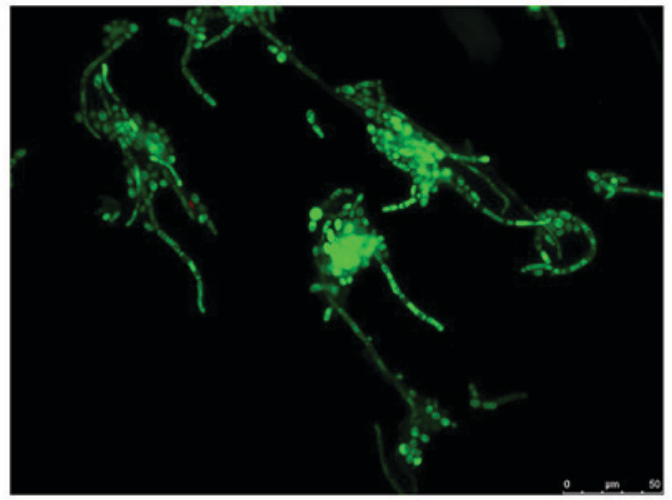

B

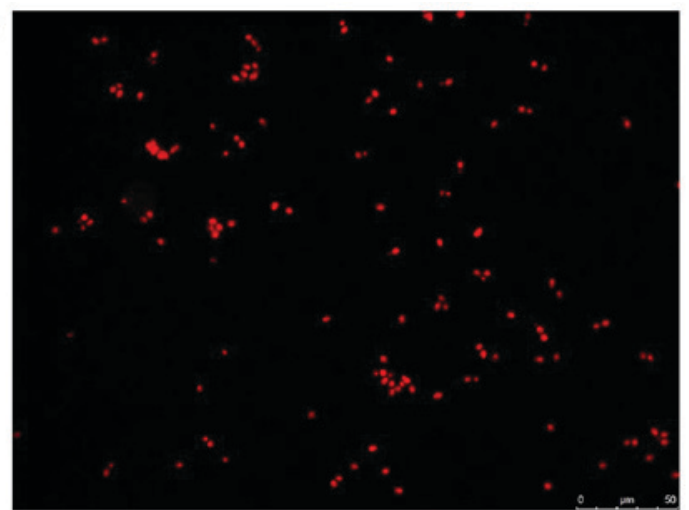

D

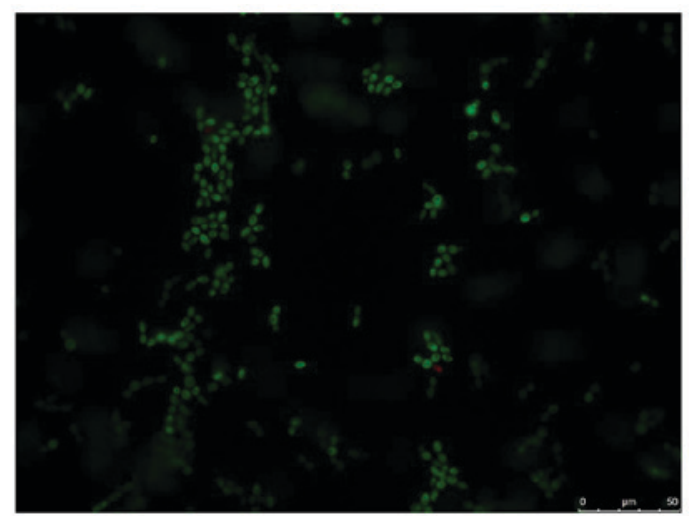

$\mathbf{F}$

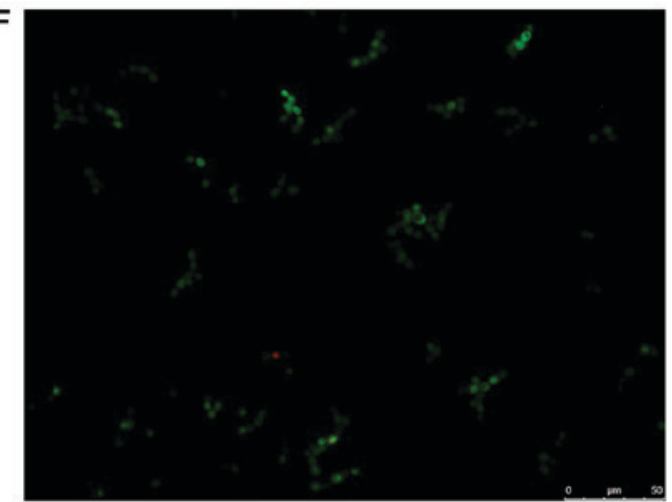

Figure 8. Viability of yeast suspension and yeast biofilm as tested by fluorescent fluorescein diacetate/ethidium bromide staining. The green staining indicates living cells and the orange staining indicates dead cells. Staining of Candida albicans ATCC 10231 suspensions following incubation at (A) $37^{\circ} \mathrm{C}$ and (B) $85^{\circ} \mathrm{C}$ for $30 \mathrm{~min}$. Staining of C. albicans ATCC 10231 biofilms harvested from the bottom of wells following incubation at $37^{\circ} \mathrm{C}$ in the $(\mathrm{C})$ absence of farnesol (control) and (D) presence of $3 \mathrm{mM}$ farnesol. Staining of C. albicans ATCC 10231 biofilms harvested from the bottom of wells following incubation at $37^{\circ} \mathrm{C}$ in the (E) absence of tyrosol (control) and (F) presence of $20 \mathrm{mM}$ tyrosol. Scale bars, $50 \mu \mathrm{m}$

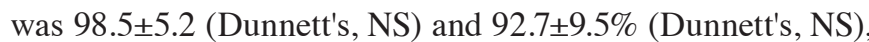
respectively. Similarly, when adding $1 \mathrm{mM}$ vs. $20 \mathrm{mM}$ tyrosol to the pre-formed biofilms, the amount of biomass attached after $24 \mathrm{~h}$ was 104.3 \pm 1.7 (Dunnett's, NS) and 76.7 $\pm 4.3 \%$ (Dunnett's, $\mathrm{P}<0.001$ ), respectively, of the paired controls, and the biomass attached after $48 \mathrm{~h}$ was $98.5 \pm 5.2$ (Dunnett's, NS) and $66.8 \pm 5.1 \%$ (Dunnett's, $\mathrm{P}<0.001$ ), respectively. The small reduction in biomass observed following the addition of $20 \mathrm{mM}$ tyrosol to the pre-formed biofilms was significant.

\section{Discussion}

The present study aimed to document the anti-biofilm effect of two QSMs (farnesol and tyrosol) at the beginning of Candida biofilm formation or to a pre-formed Candida biofilm. The effects on biofilm formation must be distinguished from those on pre-formed biofilm for considering separately the preventive and curative applications of these molecules. A recent study (26) considered the anti-biofilm effect of farnesol and tyrosol on pre-formed Candida biofilms: The authors found a significant reduction in biofilm metabolism with no effect on the biomass by using farnesol or a tyrosol/farnesol combination.

In the present study, farnesol at $3 \mathrm{mM}$ exerted a greater effect when added at the beginning of biofilm formation than when added to pre-formed biofilm. Similarly, tyrosol at $20 \mathrm{mM}$ exhibited a stronger action when added at the beginning of biofilm formation than when added to pre-formed biofilm. Despite these significant reductions in attached biomass, the yeast growth, as evaluated by turbidimetry, varied little in the 


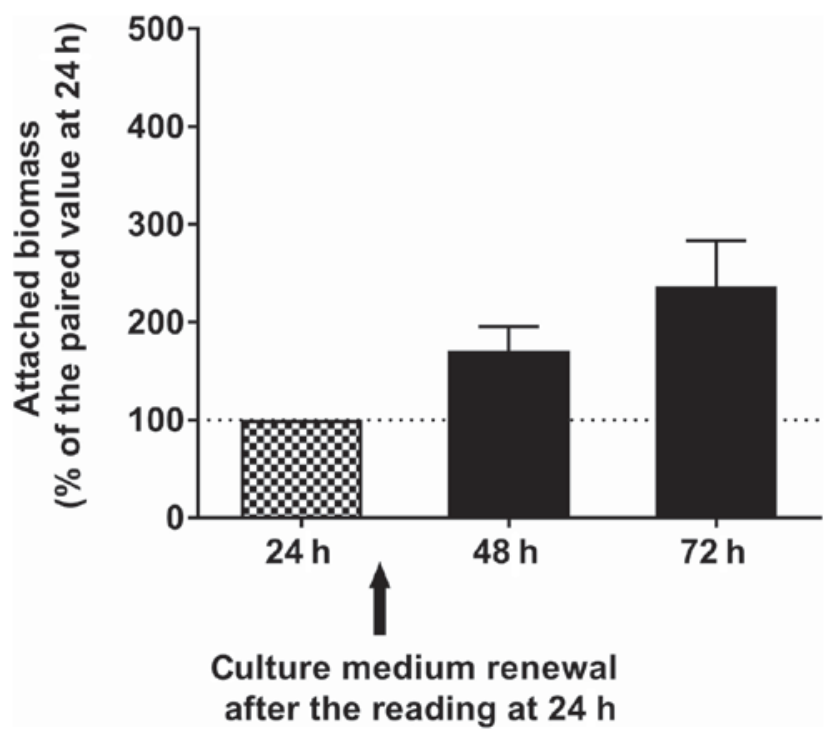

Figure 9. Time course of biofilm formation. The time course of the attached biomass (ATCC 10231) in the absence of QSMs (crystal violet staining; $\mathrm{n}=6$ ). When the $\mathrm{OD}_{600 \mathrm{~nm}}$ was $>2.000$, the well contents were diluted 10 -fold in $2 \mathrm{M}$ acetic acid. QSMs, quorum-sensing molecules.
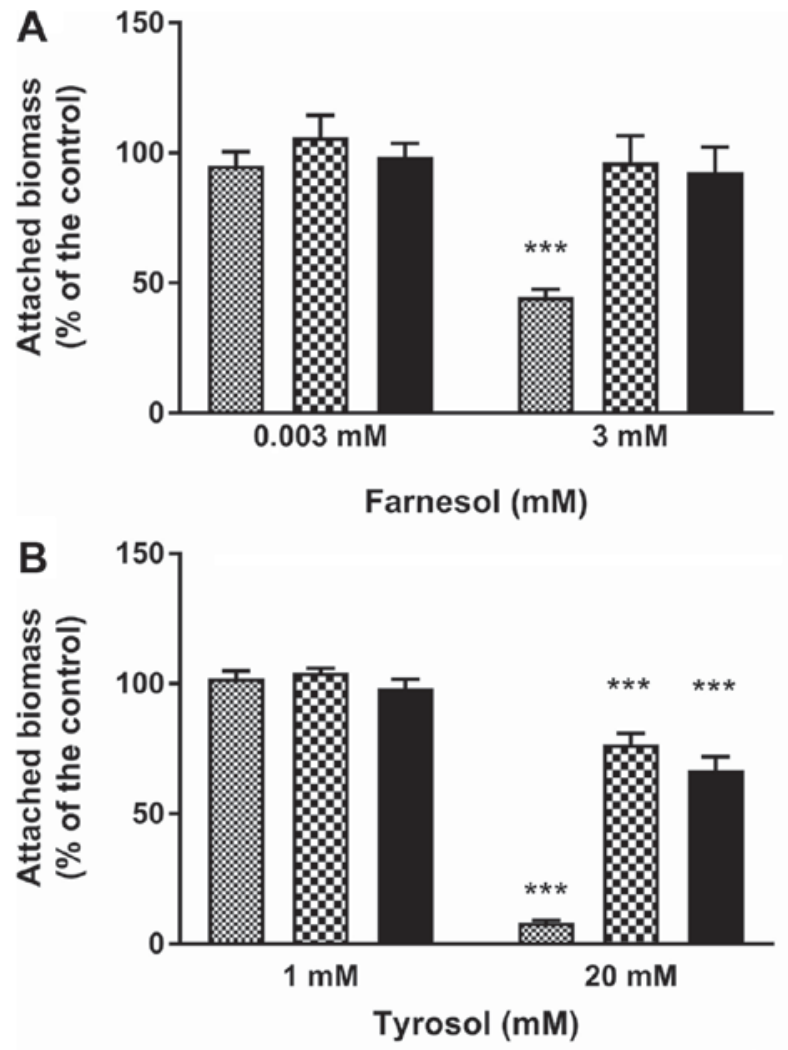

QSM effect on biofilm formation

$\mathbf{m}$ QSM effect on preformed biofilms (24 $\mathrm{h}$ incubation)

QSM effect on preformed biofilms (48 $\mathrm{h}$ incubation)

Figure 10. Effect of QSMs on pre-formed and formed Candida biofilms. The effect of (A) farnesol $(0.003$ and $3 \mathrm{mM})$ and $(\mathrm{B})$ tyrosol $(1$ and $20 \mathrm{mM})$ on biofilm production and pre-formed biofilm following 24 and $48 \mathrm{~h}$ incubations. The results are expressed as a percentage of the paired control. QSMs, quorum-sensing molecules. ${ }^{* * *} \mathrm{P}<0.001$ vs. control.

presence of the two investigated molecules. Therefore, there is an anti-biofilm effect distinct from any fungicidal or fungistatic action. The absence of fungicidal or fungistatic action observed by turbidimetry was corroborated by culturing the supernatants on solid agar followed by counting of the CFUs and by viability tests using fluorescent microscopy. These molecules, at the highest tested concentrations, had a greater effect in the initial phases of biofilm formation; however, the data do not differentiate an anti-adhesion effect from a reduction in biofilm growth. The effect of farnesol in anaerobiosis was not significantly different from that observed in aerobiosis. However, in anaerobiosis, the effect of tyrosol slightly, but significantly, decreased in comparison with that observed in aerobiosis. Notably, in the absence of both farnesol and tyrosol, there was a marked reduction in biofilm production in anaerobiosis compared to aerobiosis.

The QSM doses observed as inhibitory in vitro may not be reached in the oral environment. In culture media, the QSM concentrations quoted in the literature are in the micromolar range or lower for both farnesol (27) and tyrosol (11); however, they cannot be representative of those present in biofilms, which may concentrate these molecules. Intra-biofilm concentrations of QSMs have been scarcely investigated to date. Only one study, to the best of our knowledge, mentions a lower secretion of farnesol in Candida biofilm than in the culture medium of planktonic cells (28), and another mentions $50 \%$ higher tyrosol secretion by Candida biofilm than by planktonic cells (22). It is difficult to evaluate QSM concentrations during biofilm production as this requires harvesting of the experimental biofilm from the culture medium without modifying the intra-biofilm composition, while the biofilm itself can also behave as an open compartment with exchange between intra- and extra-biofilm areas. The concentrations of QSMs found to have an anti-biofilm effect were exogenously added in the millimolar range in the present study, which is higher than the doses generated in culture. It is therefore questionable whether the observed anti-biofilm effect is due to toxicity rather than a biological effect (27). Further studies should clarify and differentiate the metabolic pathways and cellular mechanisms involved in QS from those involved in biofilm toxicity. In this context, it would be appropriate to investigate 
the consequences of farnesol conversion to farnesoic acid with respect to biofilm formation, as previously described in C. albicans ATCC 10231 (29).

Prior to suggesting the use of these molecules for preventive purposes in an oral hygiene setting, future studies should further clarify the repercussions of these QSMs on the homeostasis of the oral microbiome. To date, few studies have addressed the effects of QSMs on oral ecology. Tyrosol suspended in saliva at a concentration above the solubility threshold has been reported to reduce the adherence of $C$. albicans and $C$. glabrata to acrylic resin, a material used in the manufacture of dentures (30). In the same study, tyrosol significantly reduced the biomass of $C$. albicans single biofilm and $C$. albicans/glabrata mixed biofilm. Farnesol at a concentration of $3.12 \mathrm{mM}$ (31) and tyrosol at concentrations of $>20 \mathrm{mM}$ (32) have been reported to inhibit the development of single and mixed biofilms formed by $C$. albicans and the cariogenic oral bacteria Streptococcus mutans. These studies suggest the development of novel oral strategies to prevent oral diseases caused by pathogenic biofilms, such as denture stomatitis and dental caries; however, tyrosol exerted no significant reduction in acid production by Candida and S. mutans (26). Some notable data have been produced concerning minor bacterial species in the oral cavity. On one hand, both tyrosol and farnesol affect certain virulence factors of the bacteria Pseudomonas aeruginosa, suggesting a possible effect of yeast QSMs in mixed biofilms when P. aeruginosa is associated with C. albicans (33). On the other hand, farnesol at a concentration of $\geq 100 \mu \mathrm{M}$ inhibits Staphylococcus aureus biofilm formation (19).

The present study demonstrates a specific anti-biofilm effect, independent of fungicidal or fungistatic action, of both farnesol and tyrosol, as tested in C. albicans ATCC 10231 and six strains isolated from dentures. This inhibition of biofilm formation by the exogenous addition of QSMs may result from a disturbance in the QSM mechanisms or from a limitation of yeast adhesion on the support; however, another toxic action on the metabolic pathways involved in biofilm formation cannot be excluded. Prior to suggesting the use of these molecules for preventive purposes in an oral hygiene setting, further studies are required to clarify the metabolic pathways, gene expression regulation and cellular mechanisms involved in QS, as well as other repercussions on the oral microbiome.

\section{Acknowledgements}

The authors would like to thank Ms. Ilhame Dardour and Mr. Hadrien Kerkhofs of the Medical Biology Department, Haute Ecole Francisco Ferrer (Brussels, Belgium) and Ms. Latifa Manouach of the Dentistry Department, Université de Bruxelles (Brussels, Belgium) for their enthusiastic participation in the study in the context of their final year dissertations. Furthermore, the authors thank Mr. G. Vegh (Laboratory of Physiology and Pharmacology, Université Libre de Bruxelles (Brussels, Belgium) for his technical help in microscope management.

\section{Funding}

The present study was supported by a grant (2013-2014) from the Xenophilia Funds (Université Libre de Bruxelles).

\section{Availability of data}

The data sets used and analysed in the current study are available from the corresponding author on reasonable request.

\section{Authors' contributions}

SS contributed to the acquisition, management and interpretation of data. ZBO validated the identification of Candida strains, collaborated in the interpretation of the data and critically revised the manuscript. PC contributed to the conception and design of this investigation; and was involved in drafting the manuscript.

\section{Ethics approval and consent to participate}

Not applicable.

\section{Patient consent for publication}

Not applicable.

\section{Competing interests}

The authors declare that they have no competing interests.

\section{References}

1. Miller MB and Bassler BL: Quorum sensing in bacteria. Annu Rev Microbiol 55: 165-199, 2001.

2. Hogan DA: Talking to themselves: Autoregulation and quorum sensing in fungi. Eukaryot Cell 5: 613-619, 2006.

3. Albuquerque $\mathrm{P}$ and Casadevall A: Quorum sensing in fungi-A review. Med Mycol 50: 337-345, 2012.

4. Cho T, Nagao JI, Imayoshi R, Kaminishi H, Aoyama T and Nakayama H: Quorum sensing and morphological regulation in the pathogenic fungus Candida albicans. J Oral Biosci 52: 233-239, 2010.

5. Kruppa M: Quorum sensing and Candida albicans. Mycoses 52: $1-10,2009$.

6. Deveau A and Hogan DA: Linking quorum sensing regulation and biofilm formation by Candida albicans. Methods Mol Biol 692: 219-233, 2011

7. Lingappa BT, Prasad M, Lingappa Y, Hunt DF and Biemann K: Phenethyl alcohol and tryptophol: Autoantibiotics produced by the fungus Candida albicans. Science 163: 192-194, 1969.

8. Hazen KC and Cutler JE: Autoregulation of germ tube formation by Candida albicans. Infect Immun 24: 661-666, 1979.

9. Hornby JM, Jensen EC, Lisec AD, Tasto JJ, Jahnke B, Shoemaker R, Dussault P and Nickerson KW: Quorum sensing in the dimorphic fungus Candida albicans is mediated by farnesol. Appl Environ Microbiol 67: 2982-2992, 2001.

10. Oh KB, Miyazawa H, Naito T and Matsuoka H: Purification and characterization of an autoregulatory substance capable of regulating the morphological transition in Candida albicans. Proc Natl Acad Sci USA 98: 4664-4668, 2001.

11. Chen H, Fujita M, Feng Q, Clardy J and Fink GR: Tyrosol is a quorum-sensing molecule in Candida albicans. Proc Natl Acad Sci USA 101: 5048-5052, 2004.

12. Westwater C, Balish E and Schofield DA: Candida albicans-conditioned medium protects yeast cells from oxidative stress: A possible link between quorum sensing and oxidative stress resistance. Eukaryot Cell 4: 1654-1661, 2005.

13. Mosel DD, Dumitru R, Hornby JM, Atkin AL and Nickerson KW: Farnesol concentrations required to block germ tube formation in Candida albicans in the presence and absence of serum. Appl Environ Microbiol 71: 4938-4940, 2005.

14. Décanis N, Tazi N, Correia A, Vilanova M and Rouabhia M: Farnesol, a fungal quorum-sensing molecule triggers Candida albicans morphological changes by downregulating the expression of different secreted aspartyl proteinase genes. Open Microbiol J 5: 119-126, 2011. 
15. Ramage G, Saville SP, Wickes BL and López-Ribot JL: Inhibition of Candida albicans biofilm formation by farnesol, a quorum-sensing molecule. Appl Environ Microbiol 68: 5459-5463, 2002.

16. Shchepin R, Hornby JM, Burger E, Niessen T, Dussault P and Nickerson KW: Quorum sensing in Candida albicans: Probing farnesol's mode of action with 40 natural and synthetic farnesol analogs. Chem Biol 10: 743-750, 2003.

17. Shareck J and Belhumeur P: Modulation of morphogenesis in Candida albicans by various small molecules. Eukaryot Cell 10: 1004-1012, 2011

18. Yu LH, Wei X, Ma M, Chen XJ and Xu SB: Possible inhibitory molecular mechanism of farnesol on the development of fluconazole resistance in Candida albicans biofilm. Antimicrob Agents Chemother 56: 770-775, 2012.

19. Jabra-Rizk MA, Shirtliff M, James C and Meiller T: Effect of farnesol on Candida dubliniensis biofilm formation and fluconazole resistance. FEMS Yeast Res 6: 1063-1073, 2006.

20. Katragkou A, McCarthy M, Alexander EL, Antachopoulos C, Meletiadis J, Jabra-Rizk MA, Petraitis V, Roilides E and Walsh TJ: In vitro interactions between farnesol and fluconazole, amphotericin B or micafungin against Candida albicans biofilms. J Antimicrob Chemother 70: 470-478, 2015.

21. Nickerson KW, Atkin AL and Hornby JM: Quorum sensing in dimorphic fungi: Farnesol and beyond. Appl Environ Microbiol 72: 3805-3813, 2006.

22. Alem MA, Oteef MD, Flowers TH and Douglas LJ: Production of tyrosol by Candida albicans biofilms and its role in quorum sensing and biofilm development. Eukaryot Cell 5: 1770-1779, 2006.

23. Cordeiro RA, Teixeira CE, Brilhante RS, Castelo-Branco DS, Alencar LP, de Oliveira JS, Monteiro AJ, Bandeira TJ, Sidrim JJ, Moreira JL and Rocha MF: Exogenous tyrosol inhibits planktonic cells and biofilms of Candida species and enhances their susceptibility to antifungals. FEMS Yeast Res 15: fov012, 2015.

24. Blanco MT, Pérez-Giraldo C, Blanco J, Morán FJ, Hurtado C and Gómez-García AC: In vitro studies of activities of some antifungal agents against Candida albicans ATCC 10231 by the turbidimetric method. Antimicrob Agents Chemother 36: 898-901, 1992.

25. Stepanović S, Vuković D, Dakić I, Savić B and Švabić-Vlahović M: A modified microtiter-plate test for quantification of staphylococcal biofilm formation. J Microbiol Methods 40: 175-179, 2000 .
26. Monteiro DR, Arias LS, Fernandes RA, Deszo da Silva LF, de Castilho MOVF, da Rosa TO, Vieira APM, Straioto FG, Barbosa DB and Delbem ACB: Antifungal activity of tyrosol and farnesol used in combination against Candida species in the planktonic state or forming biofilms. J Appl Microbiol 123: 392-400, 2017.

27. Krom BP, Levy N, Meijler MM and Jabra-Rizk MA: Farnesol and Candida albicans: Quorum sensing or not quorum sensing. Isr J Chem 56: 295-301, 2016.

28. Martins M, Henriques M, Azeredo J, Rocha SM, Coimbra MA and Oliveira R: Morphogenesis control in Candida albicans and Candida dubliniensis through signaling molecules produced by planktonic and biofilm cells. Eukaryot Cell 6: 2429-2436, 2007.

29. Riekhof WR and Nickerson KW: Quorum sensing in Candida albicans: Farnesol versus farnesoic acid. FEBS Lett 591: 1637-1640, 2017.

30. Monteiro DR, Feresin LP, Arias LS, Barão VA, Barbosa DB and Delbem ACB: Effect of tyrosol on adhesion of Candida albicans and Candida glabrata to acrylic surfaces. Med Mycol 53: 656-665, 2015.

31. Fernandes RA, Monteiro DR, Arias LS, Fernandes GL, Delbem AC and Barbosa DB: Biofilm formation by Candida albicans and Streptococcus mutans in the presence of farnesol: A quantitative evaluation. Biofouling 32: 329-338, 2016.

32. Arias LS, Delbem AC, Fernandes RA, Barbosa DB and Monteiro DR: Activity of tyrosol against single and mixed-species oral biofilms. J Appl Microbiol 120: 1240-1249, 2016.

33. Abdel-Rhmam SH, El-Mahdy AM and El-Mowafy M: Effect of tyrosol and farnesol on virulence and antibiotic resistance of clinical isolates of Pseudomonas aeruginosa. Biomed Res Int 2015: 456463, 2015.

This work is licensed under a Creative Commons Attribution-NonCommercial-NoDerivatives 4.0 International (CC BY-NC-ND 4.0) License. 